\title{
Cem dias de COVID-19 em Pernambuco, Brasil: a epidemiologia em contexto histórico
}

\author{
The first hundred days of COVID-19 in Pernambuco \\ State, Brazil: epidemiology in historical context
}

\author{
Cien días de COVID-19 en Pernambuco, Brasil: \\ la epidemiología en contexto histórico
}

Wayner Vieira de Souza 1

Celina Maria Turchi Martelli 1

Amanda Priscila de Santana Cabral Silva 1,2

Livia Teixeira de Souza Maia 2

Maria Cynthia Braga 1

Luciana Caroline Albuquerque Bezerra 3

George Santiago Dimech 3

Ulisses Ramos Montarroyos 4

Thalia Velho Barreto de Araújo 5

Demócrito de Barros Miranda-Filho 4

Ricardo Arraes de Alencar Ximenes 4,5

Maria de Fátima Pessoa Militão de Albuquerque 1

doi: 10.1590/0102-311X00228220

\section{Resumo}

A pandemia de COVID-19 iniciou sua linha do tempo em 31 de dezembro de 2019 na China e o SARS-CoV-2 identificado como agente etiológico. O objetivo deste manuscrito é descrever a dinâmica espacial e temporal da epidemia de COVID-19 nos primeiros cem dias, no Estado de Pernambuco, Brasil. Apresentamos a evolução de casos e óbitos segundo semana epidemiológica. Realizamos a análise da série do acumulado diário de casos da COVID-19 confirmados, com projeções para os 15 dias subsequentes, utilizando o aplicativo JoinPoint. Esse programa possibilita identificar pontos de inflexão testando sua significância estatística. Analisamos também a tendência de interiorização da COVID-19 no estado, considerando a distribuição percentual de casos ocorridos no Recife, municípios da Região Metropolitana de Recife e do interior, por conjuntos de três semanas, com construção de mapas temáticos. Os 100 dias da epidemia de COVID-19 resultaram em 52.213 casos e 4.235 óbitos entre 12 de março, correspondendo se 11, até 20 de junho de 2020 (semana epidemiológica 25). O pico da curva epidêmica ocorreu na semana epidemiológica 21 (23 de maio), seguido por desaceleração do número de casos. Detectou-se, inicialmente, a periferização dos casos na capital e região metropolitana, seguida por rápida disseminação para o interior do estado. Houve redução das taxas de crescimento médio diário a partir de abril, mas com patamar de mais de 6.000 casos semanais de COVID-19, em média. Ao final do período, a série de casos do estado indica persistência da circulação e transmissão comunitária do SARS-CoV-2. Finalmente, questiona-se parafraseando Garcia Marques em Cem Anos de Solidão, se estaríamos diante de "uma estiagem ou prenúncio de recrudescimento".

COVID-19; Fatores Epidemiológicos; Estudos de Séries Temporais

\author{
Correspondência \\ W. V. Souza \\ Instituto Aggeu Magalhães, Fundação Oswaldo Cruz. \\ Av. Moraes Rêgo s/n, Recife, PE 50740-465, Brasil. \\ wayner.vieira@fiocruz.br \\ 1 Instituto Aggeu Magalhães, Fundação Oswaldo Cruz, Recife, \\ Brasil. \\ 2 Universidade Federal de Pernambuco, Vitória de Santo Antão, \\ Brasil. \\ 3 Secretaria de Saúde do Estado de Pernambuco, Recife, Brasil. \\ 4 Universidade de Pernambuco, Recife, Brasil. \\ 5 Universidade Federal de Pernambuco, Recife, Brasil.
}




\section{Introdução}

A pandemia de COVID-19 que o mundo vivencia nos dias atuais iniciou sua linha do tempo em 31 de dezembro de 2019 com o aparecimento de casos de pneumonia grave de etiologia desconhecida na cidade de Wuhan, na China. Em 7 de janeiro de 2020, autoridades sanitárias chinesas identificaram o agente etiológico dessa Síndrome Respiratória Aguda Grave (SRAG) como sendo um coronavírus, o SARS-CoV-2, sendo a doença denominada COVID-19 1,2 pela Organização Mundial da Saúde (OMS).

Paralelos podem ser traçados a respeito da pandemia de gripe espanhola que acaba de completar cem anos. Num intervalo de quase um ano, entre 1918 e 1919, essa pandemia causada pela cepa do vírus $1918 \mathrm{H} 1 \mathrm{~N} 1$ causou mais de 50 milhões de mortes, mais do que a aids nos últimos 40 anos ${ }^{3}$. As referências à gripe espanhola têm sido frequentes nos últimos meses como pano de fundo para a discussão sobre a velocidade, intensidade e suficiência das medidas de controle adotadas em cada país 4 . Ashton ${ }^{4}$ enfatiza que não conhecer e aprender as lições da história nos leva a repetir os mesmos erros, dado que muito do despreparo verificado em 1918-1919 observa-se na atual pandemia. Entre os erros cometidos durante a gripe espanhola, a comunicação com a população é apontada como o principal. Como exemplo, o governo dos Estados Unidos silenciou sobre a pandemia e o lema "o medo/preocupação mata mais do que a doença" (worry kills more than the disease) era repetido à exaustão 5.

Em 3 de fevereiro de 2020, por meio da Portaria MS no 188 6, o Ministério da Saúde do Brasil declarou Emergência de Saúde Pública de Interesse Nacional (ESPIN). Tal Portaria também criou o Centro de Operações de Emergências em Saúde Pública (COE-COVID-19) como instância de gestão em âmbito nacional, sob a responsabilidade da Secretaria de Vigilância em Saúde ${ }^{7}$. A partir daí, foi construído um sistema de vigilância para registro de casos e óbitos de COVID-19, tendo também organizado a rede de laboratórios de referência.

No Brasil, em relação à pandemia de COVID-19, pode-se destacar que a velocidade de sua disseminação e o atual patamar de conhecimentos científicos geram incertezas sobre as estratégias para o enfrentamento da doença, sendo potencializadas pelas desigualdades sociais 8 . Tem-se recomendado, inclusive pela OMS, que as respostas do setor saúde deveriam ser estruturadas em etapas consonantes com a vigilância de epidemias: contenção, supressão, mitigação e recuperação 2,9.

Dentro desse contexto epidêmico, estabeleceram-se conflitos de gestão da crise sanitária, entre as lideranças de nível nacional e regionais, gerando decisão do Supremo Tribunal Federal sobre a competência de estados e municípios na formulação de medidas para o enfrentamento do novo Coronavírus, mesmo em contraposição de medidas recomendadas pelo governo federal ${ }^{10}$. Do ponto de vista de gestão em saúde, estados e municípios passaram a definir e implementar medidas de vigilância e controle de acordo com os dados epidemiológicos de suas áreas. Assim, registros de casos e óbitos da COVID-19 em níveis estadual e municipal vêm sendo objeto de análises epidemiológicas para a compreensão da dinâmica da transmissão do SARS-CoV-2 em populações específicas.

Em Pernambuco, as respostas à pandemia foram dadas rapidamente após a confirmação dos dois primeiros casos importados notificados de COVID-19 em 12 de março. Dois dias depois, o estado publicou seu primeiro Decreto Lei 11 proibindo eventos com mais de 50 pessoas, seguido por vários outros decretos, publicados no mês de março e em meses subsequentes, com medidas de distanciamento social de diversas naturezas. Entre elas, destacamos a suspensão de atividades em equipamentos culturais e em academias de ginástica, fechamento total das unidades de ensino, suspensão do comércio e serviços não essenciais, culminando com o denominado lockdown em Recife, Olinda, Camaragibe, São Lourenço da Mata e Jaboatão dos Guararapes, implantado a partir de 16 de maio, por 15 dias. Pernambuco esteve também à frente de outras intervenções não farmacêuticas ao decretar o uso obrigatório de máscaras pela população, em 16 de maio, em consonância com as evidências científicas da efetividade de seu uso na prevenção da disseminação de outras viroses respiratórias e COVID-19 12.

Chegamos aos cem dias de epidemia no estado contabilizando 52.213 casos e 4.235 óbitos devido à COVID-19, já na vigência da flexibilização do distanciamento social desde 15 de junho, mesmo na ausência de indicadores que dessem suporte a esta reabertura, conforme recomendação da OMS 13.

Sendo assim, torna-se necessário refletir sobre a transmissão dessa infecção viral em Pernambuco com o objetivo de descrever, na perspectiva social, a dinâmica espacial e temporal da epidemia de COVID-19 no estado, nos primeiros cem dias de epidemia. 


\section{Metodologia}

O local de estudo foi Pernambuco, considerando sua capital Recife, a Região Metropolitana do Recife e o interior do estado. Administrativamente, Pernambuco é dividido em 185 municípios distribuídos em 12 Regionais de Saúde, agrupadas em quatro Macrorregionais de Saúde 14. Segundo o último censo demográfico, o estado tinha uma população de 9.496.294 pessoas e sua capital contava com 1.637.834, segundo dados do Departamento de Informática do SUS (DATASUS) para 2018. A Região Metropolitana do Recife é composta por 14 municípios e concentra cerca de $42 \%$ da população do estado, segundo as mesmas projeções (DATASUS. População residente em Pernambuco. Estimativa populacional. http://tabnet.datasus.gov.br/cgi/tabcgi.exe?ibge/cnv/poptpe.def).

O estudo analisa a ocorrência da COVID-19 no estado no período de 12 de março de 2020 até 20 de junho de 2020 (término da semana epidemiológica 25), correspondendo a cem dias de epidemia.

A primeira abordagem tratou da apresentação da evolução de casos e óbitos por data de notificação, segundo semanas epidemiológicas, seguida de análise da série histórica do acumulado diário de casos confirmados da COVID-19, com projeções para os 15 dias subsequentes. Tal análise foi feita com o emprego do aplicativo JoinPoint (https://surveillance.cancer.gov/joinpoint/). Em síntese, o programa analisa dados de séries históricas buscando identificar pontos de inflexão nessas curvas. $\mathrm{O}$ programa é iniciado com o número mínimo de pontos de inflexão (por exemplo, 0 pontos, que representa uma linha reta) e testa se mais pontos de inflexão são estatisticamente significativos e devem ser adicionados ao modelo. Isso permite ao usuário testar se uma aparente mudança de tendência é estatisticamente significativa. Os testes de significância usam o método de permutação de Monte Carlo. Os modelos podem incorporar variação estimada para cada ponto (por exemplo, quando as respostas são taxas ajustadas por idade) ou utilizar um modelo de variação de Poisson, o que foi feito na presente análise. Além disso, os modelos também podem ser lineares no log da resposta $\left(\operatorname{Ln}(y)=\beta_{0}+\beta_{1} t\right)$, como aqui utilizado, para calcular a alteração da taxa percentual diária 15.

Posteriormente, procedeu-se à análise da evolução da distribuição temporal e espacial da epidemia nos bairros da capital. Nessa análise, verificou-se tal evolução segundo estratos de bairros classificados segundo condição de vida, usando indicador de renda do chefe da família, conforme descrito em Souza et al. ${ }^{16}$. Nessa estratificação, foram identificados quatro diferentes blocos considerando a renda dos chefes de família dentro de critérios do Instituto Brasileiro de Geografia e Estatística (IBGE). Os dois estratos de melhores condições socioeconômicas (A e B) congregavam 24 bairros da cidade e os outros dois estratos (C e D) agregavam 70 bairros. Os dois primeiros contavam com 306.277 habitantes e os outros dois com 1.214.302, pelos dados do último Censo Demográfico. A evolução dessa distribuição espacial do acumulado de casos na capital, bem como nos demais municípios da Região Metropolitana do Recife, também foi representada usando mapas de padrões pontuais com quatro recortes temporais (semanas 12,14, 16 e 18). A semana 18 foi a última para a qual realizou-se o georreferenciamento de casos, dado que o elevado número de casos semanais já permitia caracterizar nitidamente tal distribuição.

Por fim, verificou-se a tendência temporal de interiorização da ocorrência da COVID-19 no estado analisando-se a distribuição percentual de casos ocorridos no Recife, nos demais municípios da Região Metropolitana de Recife e nos municípios do interior em cinco recortes temporais por conjuntos de três semanas, até a semana 25. Essa marcha também foi representada por meio de mapa temático.

\section{Resultados}

Pernambuco acumulou até 20 de junho de 2020 (semana 25) 52.213 casos confirmados de COVID-19 e 4.235 óbitos. A distribuição desses casos e óbitos por semana epidemiológica é apresentada na Figura 1.

Pode-se observar que temos um pico de casos na semana 21 com decréscimo nas três semanas subsequentes, voltando a apresentar um aumento na semana 25 (14 a 20 junho) iniciada 14 dias após o relaxamento do lockdown de 15 dias em cinco municípios da Região Metropolitana de Recife a partir de 31 de maio. 


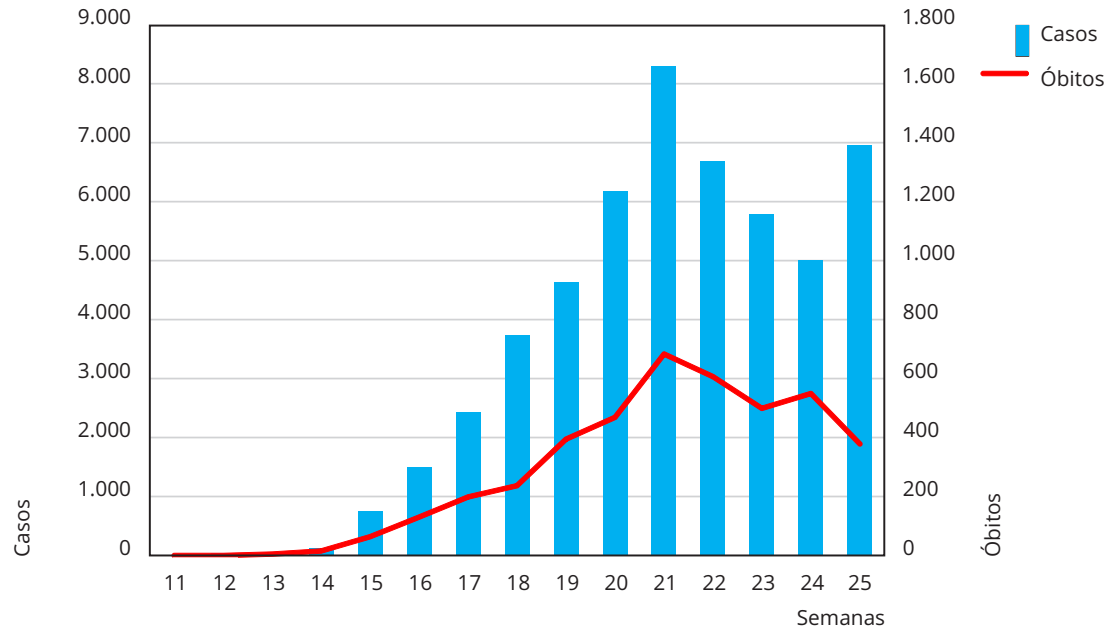

Fonte: elaborada pelos autores com base em dados da Secretaria Executiva de Vigilância em Saúde de Pernambuco.

$\mathrm{Na}$ análise da série histórica do número acumulado de casos por dia foi possível identificar cinco períodos com crescimentos significativamente diferentes. Os parâmetros dos modelos para cada trecho e a representação da série com a projeção até 5 de julho são apresentados na Figura 2.

Observe-se que nos primeiros dias de epidemia a curva de crescimento alinhou-se com um padrão de crescimento médio de cerca de $10 \%$ ao dia, o que representava aproximadamente dobrar a cada semana o número de casos. Porém, durante o mês de abril o crescimento ultrapassou largamente esse patamar crescendo em ritmo altamente preocupante em vários sentidos, principalmente em relação à capacidade instalada de leitos hospitalares. A partir do final de maio esse crescimento desacelerou passando a crescer bem abaixo daquele patamar de $10 \%$ ao dia, parâmetro usado como referência. Até o dia 20 de junho, tínhamos um acumulado de 52.213 casos, tendo o modelo predito uma ocorrência entre 48 mil e 58 mil casos aproximadamente. Para o final do período de projeção, em cinco de julho, o modelo projetou pouco mais 71 mil casos, com limites entre 64 mil e 81 mil casos. Sendo assim, a título de atualização para além dos cem dias, até essa data, o estado tinha observado mais de 65 mil casos, dentro do limite previsto pelo modelo e experimentando um patamar médio de 450 óbitos e mais de 6 mil casos nas duas semanas epidemiológicas subsequentes (semanas 26 e 27).

No acumulado de casos notificados até 22 de maio com o início dos sintomas ocorridos até a semana 18 na cidade do Recife, 8.891 casos de COVID-19 foram confirmados. Com base na referida estratificação dos bairros da cidade, observamos que no início da epidemia cerca de $40 \%$ dos casos se concentraram nos estratos C e D que representam $80 \%$ da população. Nas duas últimas semanas do período analisado, decorridas apenas seis semanas de propagação intraurbana do SARS-CoV-2, $70 \%$ dos casos já se concentravam em residentes nesses estratos, de maior vulnerabilidade social, evidenciando a acelerada periferização da transmissão viral em curto espaço de tempo, conforme apresentado na Figura 3.

A interiorização da epidemia é outro fato que também pode-se verificar ao longo do período estudado, caracterizando-se pela periferização na capital e em sua região metropolitana, progredindo com o espalhamento por todos os municípios do estado. Ao final do período, apenas em um município não havia registro de casos, fato que veio a ocorrer logo na semana seguinte. A Figura 4 ilustra esse processo territorial. 


\section{Figura 2}

Modelos de crescimento e respectivos parâmetros da Série Histórica do acumulado de casos por dia em Pernambuco, Brasil, por períodos, com projeção até 5 de julho.

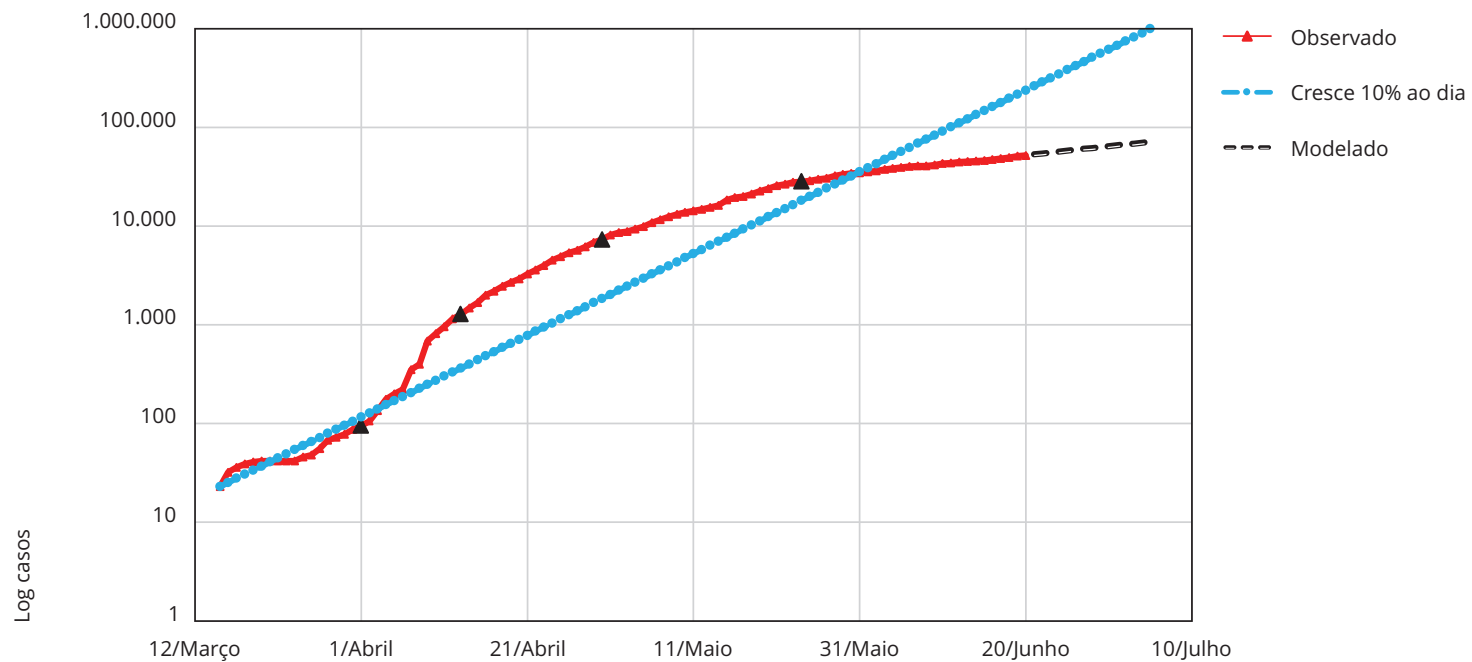

\begin{tabular}{|c|c|c|c|c|c|}
\hline Período & Intercepto & Crescimento diário \% $(\beta)$ & Erro padrão & IC95\% & Valor de $p$ \\
\hline 15/março-1/abril & 3,3206 & 6,68 & 0,0196 & $2,8-11,2$ & 0,001 \\
\hline 1/abril-13/abril & 0,4249 & 23,72 & 0,0145 & $23,2-30,5$ & $<0,001$ \\
\hline 13/abril-30/abril & 4,4963 & 9,68 & 0,0026 & $9,6-10,7$ & $<0,001$ \\
\hline 30/abril-24/maio & 6,3497 & 5,65 & 0,0007 & $5,7-6,0$ & $<0,001$ \\
\hline 24/maio-20/junho & 8,8381 & 2,09 & 0,0003 & $2,0-2,2$ & $<0,001$ \\
\hline
\end{tabular}

\section{Figura 3}

Evolução da distribuição de casos segundo períodos nos estratos de bairros do Recife, Pernambuco, Brasil, com início de sintomas até a semana epidemiológica 18.

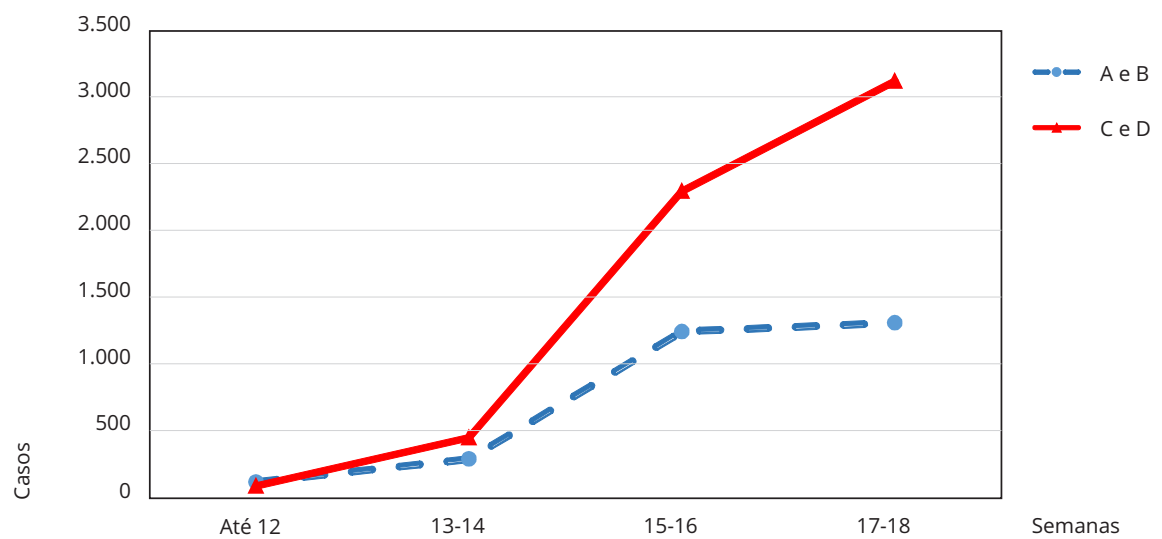

Fonte: elaborada pelos autores com base em dados da Secretaria Executiva de Vigilância em Saúde de Pernambuco. 
Figura 4

Distribuição espaço-temporal dos casos de COVID-19 na cidade de Recife, na Região Metropolitana do Recife, e no Estado de Pernambuco, Brasil.

4a) Evolução da COVID-19

segundo bairros do Recife

Semana 12

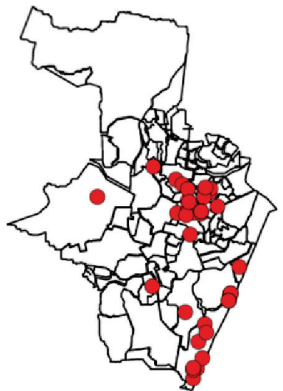

Semana 14

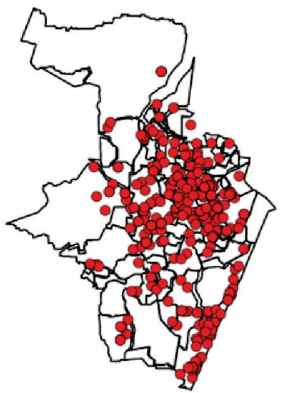

Casos de COVID-19

— Bairros

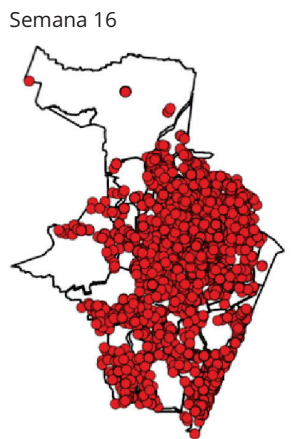

Casos de COVID-19

Bairros

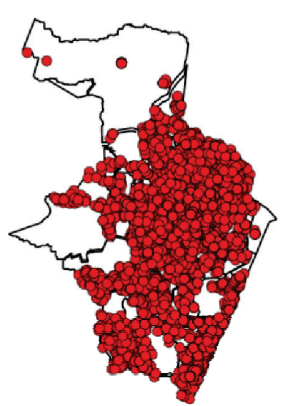

- Casos de COVID-19 4b) Evolução da COVID-19 segundo municípios da Região Metropolitana do Recife

Semana 12

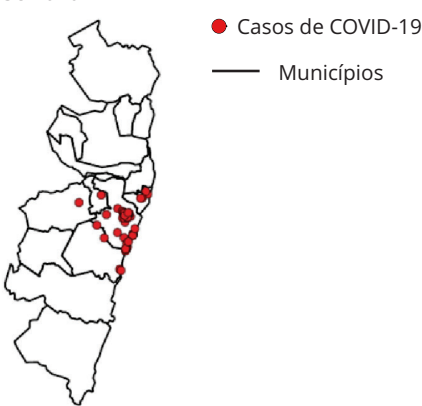

Semana 14

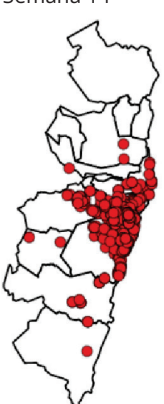

- Casos de COVID-19

— Municípios

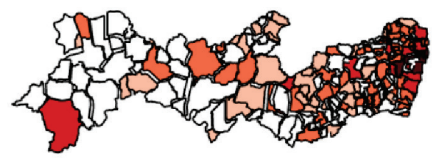

Número de casos

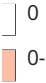

0-1

$\square^{1-10}$

10-50

50 e mais

- Municípios

Semana 16

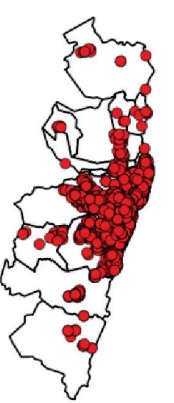

- Casos de COVID-19

— Municípios

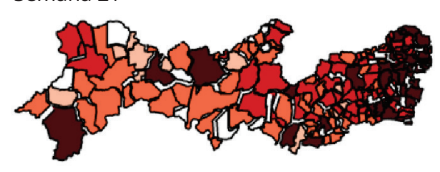

Número de casos

$\square 0$

$\bigsqcup^{0-1}$

1-10

10-50

50 e mais

- Municípios

Semana 18

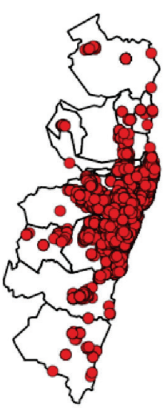

- Casos de COVID-19

— Municípios

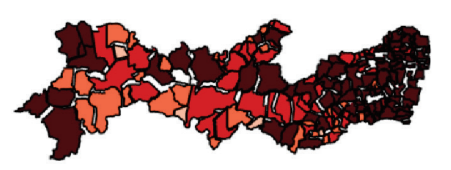

Número de casos

$\square 0$

0-1

11-10

10-50

50 e mais

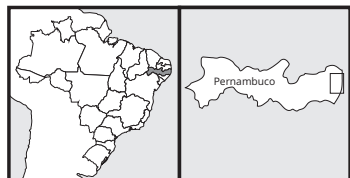


Na Tabela 1, apresenta-se em estratos de três semanas a evolução em termos quantitativos desse processo de interiorização, ressaltando-se que os municípios do interior do estado, nas últimas três semanas, já respondiam por mais da metade dos casos notificados e que somando as ocorrências nos demais municípios da Região Metropolitana de Recife reuniam 76\% dos casos do período.

Registre-se ainda que o mesmo fenômeno foi verificado com os óbitos, tendo-se contabilizado até 18 de abril (semana 16) um total de 216 óbitos, dos quais 100 (46\%) de residentes no Recife. Nas semanas posteriores (17 a 25) registraram-se 3.992 óbitos, dos quais 2.429 (61\%) de residentes em outros municípios do estado.

\section{Discussão}

Os cem dias de COVID-19 em Pernambuco resultaram em 52.213 casos confirmados e 4.235 óbitos devido à doença. A partir de 12 de março (semana epidemiológica 11), os casos confirmados de COVID-19 em Pernambuco se acumularam atingindo o pico da curva na 21 a semana (terminada em 23 de maio), quando o crescimento começou a desacelerar e o número de casos por semana epidemiológica a decrescer, embora observe-se um aumento do número de casos na última semana, terminada em 20 de junho. Verificamos durante o período, inicialmente, a periferização dos casos na capital e em sua região metropolitana, disseminando-se rapidamente em direção ao interior, atingindo quase todos os municípios do estado.

Na Região Nordeste, os estados do Maranhão, Ceará e Pernambuco inicialmente foram os principais epicentros da epidemia. No entanto, deve-se levar em conta que essa é apenas uma análise de um curto período de tempo, passível portanto de mudanças de médio e longo prazo dependendo das interações sociais e da aderência às medidas de controle.

Matos 17, em seu artigo de 2018, traz observações após cem anos da pandemia de gripe espanhola, levantando a questão se estaríamos preparados para uma nova pandemia. Conclui alertando para o padrão cíclico das pandemias de influenza e para que apesar da alta incerteza, devemos sempre ter em mente que alguma previsibilidade e que alguns ensinamentos baseados em experiências anteriores são indispensáveis, como aquelas vividas com as pandemias do século passado.

A experiência com a pandemia da COVID-19 pode ter como referência as pandemias da gripe espanhola (1918-1919), da gripe asiática (1957), da gripe de Hong Kong (1968) e da gripe suína (2009). Não obstante a magnitude e transcendência de todas, a gripe espanhola, referida por Honigsbaum 3 como a mãe de todas as pandemias, fornece elementos indispensáveis para a nossa reflexão. A gripe espanhola infectou metade da população mundial, vitimando algo próximo de 50 milhões de pessoas. O espalhamento aconteceu em ondas com intervalos de aproximadamente seis meses. A primeira delas no segundo trimestre de 1918 (primavera no hemisfério norte), a segunda, reportada como a mais severa, no último trimestre (outono) deste mesmo ano e a terceira no segundo trimestre de 1919 (primavera) 18 .

\section{Tabela 1}

Distribuição dos casos COVID-19 em Pernambuco, Brasil, segundo semanas epidemiológicas.

\begin{tabular}{|c|c|c|c|c|c|c|c|}
\hline \multirow{2}{*}{$\begin{array}{l}\text { Semanas } \\
\text { epidemiológicas }\end{array}$} & \multicolumn{2}{|c|}{ Recife } & \multicolumn{2}{|c|}{ Região Metropolitana do Recife } & \multicolumn{2}{|c|}{ Interior } & \multirow[t]{2}{*}{ Pernambuco (N) } \\
\hline & $\mathbf{n}$ & $\%$ & $\mathbf{n}$ & $\%$ & $\mathbf{n}$ & $\%$ & \\
\hline Até 13 & 46 & 73,0 & 11 & 17,5 & 6 & 9,5 & 63 \\
\hline $14-16$ & 1.209 & 57,1 & 708 & 33,4 & 202 & 9,5 & 2.119 \\
\hline $17-19$ & 5.326 & 53,4 & 3.369 & 33,8 & 1.274 & 12,8 & 9.969 \\
\hline $20-22$ & 8.584 & 40,4 & 6.612 & 31,2 & 6.026 & 28,4 & 21.222 \\
\hline $23-25$ & 4.214 & 23,9 & 4.062 & 23,0 & 9.354 & 53,1 & 17.630 \\
\hline Total & 19.379 & 38,0 & 14.762 & 28,9 & 16.862 & 33,1 & 51.003 \\
\hline
\end{tabular}

Fonte: elaborada pelos autores com base em dados da Secretaria Executiva de Vigilância em Saúde de Pernambuco. 
Tal como àquela época, não dispomos de vacina nem de tratamento específico para a doença e o que se recomenda são medidas de saúde pública em geral e de higiene pessoal, chamadas de intervenções não farmacêuticas, incluindo o distanciamento social e mesmo o isolamento de populações, visando à redução da transmissão do vírus 19.

Davies et al. 20 trazem também uma importante contribuição com análises detalhadas sobre os efeitos de diferentes intervenções, analisando também os reflexos dessas medidas na sobrecarga da demanda por leitos hospitalares, particularmente de unidade de terapia intensiva (UTI) 21 .

Segundo Horton 22, temos de empregar permanentemente medidas preventivas combinadas, contemplando as medidas de higiene pessoal, uso de máscaras, proibição de eventos de massa e o distanciamento social em geral. Essa compreensão é imprescindível e deveria ser assimilada e praticada por todos aqueles que tomam decisões nas diferentes esferas de poder. Outra constatação que o autor nos traz diz respeito ao fato de a COVID-19 não ser socialmente neutra, afetando mais fortemente os mais vulneráveis.

A pandemia de COVID-19 se depara com a população brasileira em situação vulnerável, com altas taxas de desemprego e cortes profundos nas políticas sociais. Com a aprovação da Emenda Constitucional no 95, que impõe corte radical no teto de gastos públicos e com as políticas econômicas implantadas atualmente, há um crescente e intenso estrangulamento dos investimentos em saúde e pesquisa no Brasil 8 .

Esse cenário é agravado na medida em que a restrição de circulação das pessoas influencia diretamente o acesso à renda por parte de trabalhadores informais e autônomos. O confinamento repercute diretamente em ameaças imediatas à sustentação financeira e à sobrevivência de suas famílias. A essa condição soma-se a suspensão das atividades escolares, que compromete a segurança alimentar e nutricional de crianças e adolescentes de baixa renda pelo não acesso à alimentação 23 .

Os resultados encontrados em nosso estudo também apontam na direção das populações mais vulneráveis, tendo se manifestado pela periferização das ocorrências desde os bairros centrais da capital Recife, passando para os demais municípios da Região Metropolitana do Recife e avançando para o interior do estado, onde menores recursos assistenciais estão disponíveis. Os resultados demonstram que o mapa da desigualdade traçou o caminho da epidemia.

Não obstante, há que se considerar saúde como política de estado, como ressalta a Nota Pública do Conselho Nacional de Saúde 24, de 16 de abril de 2020. Tal Nota aponta que o governo vem priorizando o discurso econômico face à questão da vida de nossa população, questão que afeta especialmente aqueles mais vulneráveis, que mais sofrem os efeitos desta conjuntura. Nesse contexto, a Nota enfatiza ainda o papel fundamental do Sistema Único de Saúde, destacando que:

“...Seguiremos exigindo, como órgão legalmente responsável pela fiscalização e monitoramento das ações do Ministério da Saúde e da Saúde Pública, que a pasta mantenha coerência com as orientações da Organização Mundial da Saúde (OMS) e dos órgãos de defesa dos direitos humanos, reafirmando a necessidade das medidas de isolamento, valorizando a ciência, a pesquisa clinica e social baseada na determinação social do processo saúde-doença" 24.

A pandemia de COVID-19 fez com que os diferentes países desenvolvessem suas estratégias para enfrentar os desafios impostos pela doença, baseados em suas situações epidemiológicas e em suas capacidades e disponibilidades de recursos. No caso chinês, foram empregadas práticas de controle que consideraram a promoção da busca ativa de casos, contemplando estratégias de supressão e contenção visando a reduzir danos à saúde e à economia, de modo a possibilitar o retorno à normalidade. Contando com o apoio da população, as políticas de controle que incluíam o relato de casos confirmados ou suspeitos em até duas horas, rastreamento e isolamento de contatos com quarentena foi possível ter eficácia na estratégia de contenção que propiciou a redução das mortes.

A incorporação das chamadas intervenções não farmacêuticas serviu para fortalecer as medidas no âmbito da vigilância epidemiológica, ampliando a capacidade de redução da incidência de casos. Tais intervenções de distanciamento social contemplaram a suspensão do transporte público, fechamento de locais de diversão e a proibição de eventos de massa. Registre-se ainda que em períodos de relaxamento do distanciamento, a busca ativa de casos e a vigilância de contatos deve ser mantida para garantir o controle da transmissão comunitária. Como apontam Ferguson et al. ${ }^{19}$, o relaxamento das medidas de distanciamento social baseado em tendências dos dados de vigilância de períodos curtos deve ser revisto caso se verifique novo aumento do número de casos 8,19. 
Não obstante termos evidências de que a partir de 13 de abril as taxas de crescimento médio diário do número acumulado de casos foram decrescentes, pela técnica de análise da série por regressões locais, o patamar de mais de 6 mil casos semanais de COVID-19 em Pernambuco, em média, não nos permite relaxar a vigilância. Ao final do período de cem dias a série de casos do estado apresentou um crescimento estável, porém com números de casos detectados ainda altos. Ainda que as notificações apontem que mais de $90 \%$ desses casos são leves ou moderados, que não demandam hospitalização, que poderiam ser explicados pelo aumento da testagem dos suspeitos e sintomáticos (Secretaria de Planejamento e Gestão. COVID-19 em dados. https://dados.seplag.pe.gov.br/apps/corona.html\# testes). Entretanto, essa alta média semanal indica persistência da circulação do SARS-CoV-2 em nosso meio.

A discussão sobre a importância de perseverar na vigilância exige uma visão com foco aberto para entendermos realmente a fase da pandemia em que nos encontramos. E essa necessidade, aliada à constatação de que a COVID-19 afeta mais os vulneráveis, traz à memória um trecho de Cem Anos de Solidão 25 (p. 274), uma das mais importantes obras da literatura latino-americana:

"Choveu durante quatro anos, onze meses e dois dias. Houve épocas de chuvisco em que todo mundo pôs a sua roupa de domingo e compôs uma cara de convalescente para festejar a estiagem, mas logo se acostumaram a interpretar as pausas como anúncio de recrudescimento".

A tempestade passou ou estamos numa estiagem?

\section{Colaboradores}

W. V. Souza, C. M. Turchi Martelli, A. P. S. C. Silva e M. F. P. M. Albuquerque contribuíram na concepção, redação, análise e discussão dos resultados. L. T. S. Maia, M. C. Braga, L. C. A. Bezerra, G. S. Dimech, U. R. Montarroyos, T. V. B. Araújo, D. B. MirandaFilho e R. A. A. Ximenes contribuíram na revisão do texto, análise e discussão dos resultados.

\section{Informações adicionais}

ORCID: Wayner Vieira de Souza (0000-0002-09399332); Celina Maria Turchi Martelli (0000-00022491-0688); Amanda Priscila de Santana Cabral Silva (0000-0003-2337-9925); Lívia Teixeira de Souza Maia (0000-0002-0161-7729); Maria Cynthia Braga (0000-0002-7862-6455); Luciana Caroline Albuquerque Bezerra (0000-0001-6074-6072); George Santiago Dimech (0000-0003-0295-3393); Ulisses Ramos Montarroyos (0000-0001-89675693); Thalia Velho Barreto de Araújo (0000-00019956-4145); Demócrito de Barros Miranda-Filho (0000-0003-2537-1476); Ricardo Arraes de Alencar Ximenes (0000-0002-9951-8840); Maria de Fátima Pessoa Militão de Albuquerque (0000-0002-49994160).

\section{Agradecimentos}

Agradecemos à Secretaria Estadual de Saúde de Pernambuco pela constante disponibilização dos dados que permitiram a construção destas análises. Os autores receberam apoio parcial do Conselho Nacional de Desenvolvimento Científico e Tecnológico - CNPq (bolsa de estudos 308974/20182 para C. M. T. Martelli, 309722/2017-9 para R. A. A. Ximenes, 301905/2017-7 para M. F. P. M. Albuquerque, 303953/2018-7 para M. C. Braga e 303661/2017-8 para W. V. Souza). 


\section{Referências}

1. Sohrabi C, Alsafi Z, O’Neill N, Khan M, Kerwan A, Al-Jabir A, et al. World Health Organization declares global emergency: a review of the 2019 novel coronavirus (COVID-19). Int J Surg 2020; 76:71-6.

2. World Health Organization. Clinical management of severe acute respiratory infection when novel coronavirus $(2019-\mathrm{nCoV})$ infection is suspected: interim guidance. Geneva: World Health Organization; 2020.

3. Honigsbaum M. Spanish influenza redux: revisiting the mother of all pandemics. Lancet 2018; 391:2492-5.

4. Ashton J. COVID-19 and the 'Spanish' flu. J R Soc Med 2020; 113:197-8.

5. Barry JM. Pandemics: avoiding the mistakes of 1918. Nature 2009; 459:324-5.

6. Ministério da Saúde. Portaria no 188, de 3 de fevereiro de 2020. Declara Emergência em Saúde Pública de importância Nacional (ESPIN) em decorrência da Infecção Humana pelo novo Coronavírus (2019-nCoV). Diário Oficial da União 2020; 4 fev.

7. Ministério da Saúde. Plano de Contingência Nacional para Infecção Humana pelo novo Coronavírus COVID-19. https://portalar quivos2.saude.gov.br/images/pdf/2020/fe vereiro/13/plano-contingencia-coronavirusCOVID19.pdf (acessado em 24/Jul/2020).

8. Werneck GL, Carvalho MS. A pandemia de COVID-19 no Brasil: crônica de uma crise sanitária anunciada. Cad Saúde Pública 2020; 36:e00068820.

9. Li Z, Chen Q, Feng L, Rodewald L, Xia Y, Yu $\mathrm{H}$, et al. Active case finding with case management: the key to tackling the COVID-19 pandemic. Lancet 2020; 396:63-70.

10. Supremo Tribunal Federal. Ação Direta de Inconstitucionalidade (ADI) no 6.341. http:// portal.stf.jus.br/processos/detalhe.asp?inci dente $=5880765$ (acessado em 24/Jul/2020).

11. Governo do Estado de Pernambuco. Decreto no 48.809, de 14 de março de 2020. Regulamenta, no Estado de Pernambuco, medidas temporárias para enfrentamento da emergência de saúde pública de importância internacional decorrente do coronavírus, conforme previsto na Lei Federal no 13.979, de 6 de fevereiro de 2020. Diário Oficial do Estado de Pernambuco 2020; 14 mar.

12. Chu DK, Akl EA, Duda S, Solo K, Yaacoub S, Schünemann HJ, et al. Physical distancing, face masks, and eye protection to prevent personto-person transmission of SARS-CoV-2 and COVID-19: a systematic review and metaanalysis. Lancet 2020; 395:1973-87.

13. World Health Organization. Coronavirus disease 2019 (COVID-19) Situation Report - 80. Geneva: World Health Organization; 2020.
14. Secretaria Estadual de Saúde, Governo do Estado de Pernambuco. Plano Diretor de Regionalização. http://portal.saude.pe.gov.br/sites/ portal.saude.pe.gov.br/files/pdrconass-ver sao_final1.doc_ao_conass_em_jan_2012.pdf (acessado em 24/Jul/2020).

15. Kim H-J, Fay MP, Feuer EJ, Midthune DN. Permutation tests for joinpoint regression with applications to cancer rates. Stat Med 2000; 19:335-51.

16. Souza WV, Albuquerque MFPM, Vazquez E, Bezerra LCA, Mendes ACG, Lyra TM, et al. Microcephaly epidemic related to the Zika virus and living conditions in Recife, Northeast Brazil. BMC Public Health 2018; 18:130.

17. Matos HJ. A próxima pandemia: estamos preparados? Revista Pan-Amazônica de Saúde 2018; 9:9-11.

18. Akin L. Understanding dynamics of pandemics. Turk J Med Sci 2020; 50:515-9.

19. Ferguson NM, Laydon D, Nedjati-Gilani G, Imai N, Ainslie K, Baguelin M, et al. Impact of non-pharmaceutical interventions (NPIs) to reduce COVID-19 mortality and healthcare demand. Imperial College COVID-19 Response Team 2020; 16 mar. https://spiral. imperial.ac.uk:8443/handle/10044/1/77482.

20. Davies NG, Kucharski AJ, Eggo RM, Gimma A, Edmunds WJ; Centre for the Mathematical Modelling of Infectious Diseases COVID-19 working group. Effects of non-pharmaceutical interventions on COVID-19 cases, deaths, and demand for hospital services in the UK: a modelling study. Lancet Public Health 2020; 5:e375-e5.

21. Colbourn T. Unlocking UK COVID-19 policy. Lancet Public Health 2020; 5:e362-3.

22. Horton R. Offline: the second wave. Lancet 2020; 395:1960.

23. Pires RR. Os efeitos sobre grupos sociais e territórios vulnerabilizados das medidas de enfrentamento à crise sanitária da COVID-19: propostas para o aperfeiçoamento da ação pública. (Nota técnica 33). http://repositorio. ipea.gov.br/handle/11058/9839 (acessado em 24/Jul/2020).

24. Conselho Nacional de Saúde. CNS considera irresponsável mudanças na pasta da saúde em meio à pandemia do Covid-19. https:// conselho.saude.gov.br/ultimas-noticiascns/1123-nota-publica-cns-considera-irres ponsavel-mudancas-na-pasta-da-saude-emmeio-a-pandemia-do-covid-19 (acessado em 24/Jul/2020).

25. Marquéz GG. Cien años de soledad. Buenos Aires: Editora Editorial Sudamericana; 1967. 
Abstract

The timeline of the COVID-19 pandemic began on December 31, 2019, in China, with SARS-CoV-2 identified as the etiological agent. This article aims to describe the COVID-19 epidemic's spatial and temporal dynamics in the first hundred days in the state of Pernambuco, Brazil. We present the evolution in cases and deaths according to epidemiological weeks. We analyzed the series of accumulated daily confirmed COVID-19 cases, with projections for the subsequent 15 days, using the JoinPoint app. This software allows identifying turning points, testing their statistical significance. We also analyze the trend in the spread of COVID-19 to the interior of the state, considering the percent distribution of cases in the State capital, Recife, municipalities in Greater Metropolitan Recife, and the state's interior, by sets of three weeks, constructing thematic maps. The first hundred days of the COVID-19 epidemic resulted in 52,213 cases and 4,235 deaths from March 12, or epidemiological week 11, until June 20, 2020 (epidemiological week 25). The peak in the epidemic curve occurred in epidemiological week 21 (May 23), followed by deceleration in the number of cases. We initially detected the spread of cases from the city center to the periphery of the state capital and Metropolitan Area, followed by rapid spread to the state's interior. There was a decrease in the mean daily growth starting in April, but with an average threshold of more than 6,000 weekly cases of COVID-19. At the end of the period, the state's case series indicates the persistence of SARS-CoV-2 circulation and community transmission. Finally, paraphrasing Gabriel Garcia Marques in One Hundred Years of Solitude, we ask whether we are facing "a pause in the storm or a sign of redoubled rain".

COVID-19; Epidemiologic Factors; Time Series Studies

\section{Resumen}

La pandemia de COVID-19 inicia su línea del tiempo el 31 de dicembre de 2019 en China y el SARS-CoV-2 identificado como agente etiológico. El objetivo de este trabajo original es describir la dinámica espacial y temporal de la epidemia de COVID-19 en los primeros cien días de epidemia, en el estado de Pernambuco, Brasil. Presentamos la evolución de casos y óbitos según las semanas epidemiológicas. Realizamos el análisis de la serie del acumulado diario de casos de COVID-19 confirmados, con proyecciones para los 15 días subsiguientes, utilizándose la aplicación JoinPoint. Este programa posibilita identificar puntos de inflexión, probando su significancia estadística. Analizamos también la tendencia de interiorización de la COVID-19 en el estado, considerándose la distribución porcentual de casos ocurridos en Recife, municipios de la Región Metropolitana de Recife y del interior, por conjuntos de tres semanas, con unas construcciones de mapas temáticos. Los cien días de la epidemia de COVID-19 resultaron en 52.213 casos y 4.235 óbitos entre el 12 de marzo, correspondiendo a la semana epidemiológica 11, hasta el 20 de juno de 2020 (semana epidemiológica 25). El pico de la curva epidémica ocurrió en la semana epidemiológica 21 (23 de mayo), seguido de una desaceleración en el número de casos. Se detectó, inicialmente, la periferización de los casos en la capital y región metropolitana, seguido por la rápida diseminación hacia el interior del estado. Hubo una reducción de las tasas de crecimiento medio diario a partir de abril, pero con un nivel de más de 6.000 casos semanales de COVID-19 de media. Al final del período la serie de casos del estado indica la persistencia de la circulación y transmisión comunitaria del SARS-CoV-2. Finalmente, se cuestiona, parafraseando a García Márquez en Cien Años de Soledad, si estamos ante "un periodo de remisión o la antesala de un recrudecimiento".

COVID-19; Factores Epidemiológicos; Estudios

de Series Temporales
Recebido em 03/Ago/2020

Versão final reapresentada em 13/Ago/2020

Aprovado em 20/Ago/2020 